\title{
QUALIDADE SANITÁRIA E GERMINAÇÃO DE SEMENTES DE PINHÃO-MANSO ${ }^{1}$
}

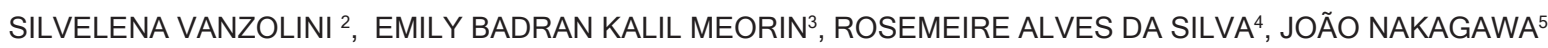

\begin{abstract}
RESUMO - O pinhão-manso é uma espécie arbustiva que está se destacando pelo interesse para produção de biocombustível, porém, os estudos sobre controle de qualidade das sementes estão se iniciando. Neste trabalho objetivou-se estudar o efeito da temperatura e do tempo de contagem na germinação de sementes de pinhão-manso, conduzido em papel toalha bem como avaliar a qualidade sanitária das sementes, desinfestadas ou não com hipoclorito de sódio, a $2 \%$. O estudo foi conduzido, com sementes de pinhão-manso extraídas de frutos maduros, coletados na planta. $\mathrm{O}$ teste de germinação foi conduzido nas temperaturas de $25{ }^{\circ} \mathrm{C}$ e alternadas de $20-30{ }^{\circ} \mathrm{C}$, com contagens aos 6,9 e 12 dias. $\mathrm{O}$ teste de sanidade foi conduzido a $20^{\circ} \mathrm{C}$ com fotoperíodo de $12 \mathrm{~h}$ por 7 dias. Foi utilizado o delineamento experimental inteiramente casualizado com quatro repetições e empregado o teste F na análise de variância dos dados de germinação e do índice de velocidade de germinação. As médias foram comparadas pelo teste de Tukey a 5\% de probabilidade. O potencial máximo de germinação das sementes de pinhão-manso foi obtido na temperatura de $25^{\circ} \mathrm{C}$, com contagens aos seis, nove e 12 dias, entretanto não apresentou diferença significativa com as temperaturas de $20-30{ }^{\circ} \mathrm{C}$, nas contagens aos 9 e 12 dias. As sementes de pinhão-manso analisadas apresentaram alta frequência de espécies fúngicas, independente da desinfestação superficial. A incidência de fungos em ordem decrescente foi dos gêneros: Alternaria, Macrophomina, Cladosporium, Colletotrichum, Fusarium, Pestalotiopsis, Phoma, Helminthosporium, Epicocum e Nigrospora a maioria destes fungos apresenta potencial patogênico.
\end{abstract}

Termos para indexação: sanidade, fungos, teste de germinação, Jatropha curcas L.

\section{HEALTH QUALITY AND GERMINATION OF Jatropha curcas SEEDS}

\begin{abstract}
Jatropha curcas is a shrubby species, interesting for biofuel production, however studies of these seeds quality control are starting. The work aimed to study effects of temperature and counting time on germination of $J$. curcas, in paper towel and to check the health quality of seeds, disinfected or not with sodium hypochlorite $2 \%$. The tests carried out with a lot of $J$. curcas seeds extracted four yellow fruits, collected in the plant. The seed germination was tested at temperature of $25^{\circ} \mathrm{C}$ and in the alternating temperature of 20 to $30^{\circ} \mathrm{C}$, with couting times at six, nine and 12 days after sowing. We used a randomized experimental design with four replications and used the $\mathrm{F}$ test for the analysis of variance of germination and index of germination speed. Means were compared by Tukey test at 5\% probability. The maximum germination potential of
\end{abstract}

\footnotetext{
${ }^{1}$ Submetido em 11/01/2010. Aceito para publicação em 01/06/2010. Monografia de graduação da segunda autora.

${ }^{2}$ Eng. Agr., Dr. Professora, Fundação Educacional de Ituverava-FEI, FAFRAM. Km 1, Cx.P.: 111, CEP: 14500-000 Ituverava/SP, e-mail: sv.segato@bol.com.br.
}

\footnotetext{
${ }^{3}$ Aluno do curso de Agronomia. FEI - FAFRAM.

${ }^{4}$ Aluno do curso de Biologia. FEI - FAFRAM.

${ }^{5}$ Eng. Agr., Professor Titular Aposentado, Voluntário, Departamento de Produção Vegetal, UNESP/Botucatu. Caixa Postal 237, CEP: 18610-307, Botucatu, SP, secdamv@fca.unesp.br.
} 
the seeds of $J$. curcas was got at $25^{\circ} \mathrm{C}$, with counts at 6,9 and 12 days, however no had significant difference in temperature of $20-30{ }^{\circ} \mathrm{C}$ with score at 9 and 12 days. The seeds of Jatropha curcas analyzed showed high frequency of fungal species independent of surface disinfection. The incidence of fungi, in decreased sequence was to the genera: Alternaria, Macrophomina, Cladosporium, Colletotrichum, Fusarium, Pestalotiopsis, Phoma, Helminthosporium, Epicocum and Nigrospora most of these fungi have pathogenic potential.

Index terms: sanity, fungus, germination test, Jatropha curcas L.

\section{INTRODUÇÃO}

O pinhão-manso (Jatropha curcas L.), arbusto, que pode atingir até quatro metros de altura, pertence à família Euphorbiaceae, sendo amplamente distribuído em áreas tropicais e subtropicais, e apresentando potencial para a produção de biocombustível (Sujatha et al., 2008). Essa espécie ocorre espontaneamente desde o Maranhão até o Paraná; é resistente à seca e apresenta crescimento mais rápido em regiões de clima quente (Arruda et al., 2004).

No Brasil o pinhão-manso vem se destacando como uma espécie promissora para a produção de biocombustível a partir de suas sementes (Saturnino et al., 2005). De acordo ainda com os mesmos autores, estas são constituídas por um tegumento denso e escuro, que envolve o embrião, o qual é rico em um óleo inodoro que queima sem emitir fumaça.

Devido ao aumento de interesse por esta espécie, verifica-se uma crescente demanda por sementes de pinhãomanso de boa qualidade fisiológica. Nesse contexto, é fundamental observar a sanidade das mesmas, pois vários são os danos que podem ser provocados por patógenos associados às sementes, dentre estes a morte em préemergência, podridão radicular, tombamento de mudas, manchas necróticas em folhas, caules, frutos, deformações como hipertrofias e subdesenvolvimento, descoloração de tecidos e infecções latentes (Neergaard, 1979). Outros danos podem ser provocados na própria semente, como podridão e perda do poder germinativo. Desta forma, para culturas agronômicas, esses danos resultam em redução do estande e da produtividade (Machado, 1988).

Em sementes desinfestadas de pinhão-manso Melo et al. (2007) observaram incidência de fungos pertencentes aos gêneros Monilia sp. (37\%), Fusarium sp. (19\%), Rhizopus sp., Trichoderma sp. (13\%), Aspergillus spp. 12\%), Curvularia sp. (5\%), Alternaria spp. (4\%) e,
Chaetomium sp. e Penicillium sp. (2\%). Por outro lado em sementes sem desinfestação, os autores observaram fungos como Aspergillus sp. (57\%), Fusarium sp. (39\%), Colletotrichum sp. (31\%), Rhizopus sp. (27\%), Monilia sp. (24\%), Alternaria sp. (16\%) e, Penicillium sp. (6\%).

O teste de germinação é a principal variável utilizada para a determinação da qualidade fisiológica das sementes e permite conhecer o potencial germinativo de um lote em condições de ambiente favoráveis (Carvalho e Nakagawa, 2000). Para tanto, o teste deve seguir um procedimento padrão recomendado pelas RAS - Regras para Análise de Sementes, publicação oficial que normatiza a análise de sementes, para que seja reproduzível, e a germinação ocorra nas condições ótimas para cada espécie. No entanto, para o pinhão-manso, a metodologia do teste ainda não foi estabelecida (Brasil, 2009).

Recentemente, alguns estudos vêm sendo direcionados para este tema, mas os resultados são, ainda, pouco conclusivos. Segundo Dantas et al. (2009), para a avaliação da germinação de sementes de pinhão-manso em laboratório, devem-se semear as mesmas em rolo de papel toalha e mantê-las em germinador a $25^{\circ} \mathrm{C}$, enquanto Martins et al.(2008) sugerem usar as temperaturas alternadas $20-30{ }^{\circ} \mathrm{C}$ em substrato de areia ou papel e com contagem final aos 10 dias da semeadura.

$\mathrm{Na}$ ausência de um protocolo para o teste de germinação de sementes de pinhão-manso o usual é testar as condições indicadas para espécies de mesmo gênero ou família. A mamoneira (Ricinus communis L.), pertencente à mesma família (Euphorbiaceae) do pinhãomanso, também apresenta sementes com alto teor de óleo e é uma espécie originária de clima tropical (Beltrão et al., 2001). Neste sentido torna-se recomendável avaliar o protocolo da RAS (Brasil, 1992) desta espécie para o teste de germinação de sementes de pinhão-manso.

Assim, neste trabalho visou-se estudar o efeito da 
temperatura e do tempo de contagem na germinação de sementes de pinhão-manso (Jatropha curcas L.), conduzido em papel toalha, bem como verificar a qualidade sanitária de sementes recém-colhidas.

\section{MATERIAL E MÉTODOS}

O experimento foi realizado no Laboratório de Análise de Sementes da Faculdade de Agronomia "Dr. Francisco Maeda", em Ituverava, SP. Sendo conduzido com sementes de pinhão-manso provenientes de frutos amarelos, coletados manualmente em 29/05/2009 de plantas com três anos de idade, localizadas no Centro Universitário Moura Lacerda, em Ribeirão Preto, SP. As sementes foram extraídas dos frutos no mesmo dia da coleta, também manualmente, colocadas em recipiente fechado e mantidas por uma semana dentro de uma câmara regulada previamente a $10{ }^{\circ} \mathrm{C}$.

$\mathrm{O}$ teor de água das sementes foi determinado pelo método da estufa, a $105{ }^{\circ} \mathrm{C} \pm 3{ }^{\circ} \mathrm{C}$ por $24 \mathrm{~h}$ (Brasil, 1992), imediatamente antes da instalação dos testes de germinação e sanidade, utilizando-se três repetições de 25 sementes.

O lote de semente assim obtido foi avaliado quanto à qualidade fisiológica e sanitária utilizando as seguintes metodologias: a) Teste de germinação: foi conduzido com as temperaturas alternadas $20-30{ }^{\circ} \mathrm{C}$ (recomendadas para sementes de mamoneira) e a constante de $25^{\circ} \mathrm{C}$ (mais comum para a maioria das espécies vegetais). Para cada teste foram utilizadas 200 sementes (quatro subamostras de 50), sem desinfestação, semeadas em rolos de papel toalha, os quais foram umedecidos com água equivalente a 2,5 vezes a massa do substrato seco. As avaliações foram realizadas ao sexto, nono e décimo segundo dia após a semeadura. Os resultados foram expressos em porcentagem de plântulas normais (Brasil, 1992). b) Índice de velocidade de germinação de plântulas: foi conduzido em conjunto com o teste de germinação, anotando-se diariamente, o número de sementes germinadas. Ao final do teste, com os dados diários do número de sementes germinadas, foi calculado o IVG empregando-se a fórmula proposta por Maguire (1962). c) Qualidade sanitária: a amostra de sementes foi dividida em duas frações: uma delas foi desinfestada com hipoclorito de sódio a $2 \%$ durante um minuto; a outra foi submetida à lavagem durante um minuto com água destilada. A avaliação da sanidade foi realizada, utilizando-se o método do papel de filtro (Brasil, 1992). Foram avaliadas 200 sementes de cada fração, as quais foram subdivididas em vinte repetições de 10 sementes. Foram utilizadas caixas de acrílico tipo gerbox previamente desinfestadas com álcool $70 \%$. Cada gerbox foi forrada com três folhas de papel de filtro esterilizadas e pré-umedecidas com água destilada. Após os sete dias de incubação, sob temperatura de $20 \pm 2$ ${ }^{\circ} \mathrm{C}$ e luz fluorescente branca com fotoperíodo de 12 horas foram feitas as análises das sementes, individualmente, ao microscópio estereoscópico verificando-se a incidência de fungos, e quando necessário foi realizado exame ao microscópio de luz para sua identificação, comparando com informações disponíveis na literatura (Barnett e Hunter, 1987).

Foi utilizado o delineamento experimental inteiramente casualizado e empregado o teste $\mathrm{F}$ para a análise de variância dos dados. Para a germinação, os dados foram analisados em esquema de parcelas subdivididas, sendo as datas de contagens consideradas as parcelas e as temperaturas as subparcelas. As médias referentes ao teste de germinação e índice de velocidade de germinação foram comparadas pelo teste de Tukey a $5 \%$ de probabilidade. Os resultados de sanidade não foram analisados estatisticamente.

\section{RESULTADOS E DISCUSSÃO}

As sementes de pinhão-manso por ocasião das análises apresentavam $12,5 \%$ de teor de água, podendo considerá-las secas.

Na Tabela 1 encontram-se os dados médios referentes à germinação das sementes. Apenas na primeira contagem da germinação houve diferença significativa, favorável à temperatura constante de $25{ }^{\circ} \mathrm{C}$ que promoveu maior número de plântulas normais aos seis dias após a semeadura em relação à temperatura alternada de 20$30{ }^{\circ} \mathrm{C}$. Na contagem aos nove e pelo IVG, a tendência de maior germinação e de velocidade de germinação na temperatura de $25{ }^{\circ} \mathrm{C}$ foi mantida, porém sem diferença significativa; apenas aos 12 dias após a semeadura a temperatura alternada promoveu maior valor numérico, mas não diferiu da temperatura de $25{ }^{\circ} \mathrm{C}$. Para ambas as temperaturas, as porcentagens de germinação foram semelhantes nas contagens realizadas aos nove e 12 dias. Entretanto, a $25{ }^{\circ} \mathrm{C}$, somente aos 12 dias foi observada diferença significativa em relação à primeira contagem realizada aos seis dias. Pelos resultados, é de 12 dias o período mais adequado para avaliar a germinação de sementes de pinhão-manso. 
TABELA 1. Dados médios de germinação (\%) aos 6,9 e 12 dias após a semeadura (DAS) e índice de velocidade de germinação (IVG) de sementes de pinhão-manso (Jatropha curcas L.) em diferentes temperaturas.

\begin{tabular}{lcrrr}
\hline \multirow{2}{*}{ Temperaturas } & \multicolumn{3}{c}{ Contagens da germinação (\%) } & \multirow{2}{*}{ IVG } \\
\cline { 2 - 4 } & $6 \mathrm{DAS}$ & $9 \mathrm{DAS}$ & $12 \mathrm{DAS}$ & \\
\hline $\mathrm{T}_{1\left(20-30^{\circ} \mathrm{C}\right)}$ & $49,00 \mathrm{~B} \mathrm{~b}$ & $79,00 \mathrm{~A} \mathrm{a}$ & $87,00 \mathrm{~A} \mathrm{a}$ & $6,08 \mathrm{~A}$ \\
$\mathrm{~T}_{2\left(25^{\circ} \mathrm{C}\right)}$ & $65,50 \mathrm{~A} \mathrm{~b}$ & $81,00 \mathrm{~A} \mathrm{ab}$ & $84,00 \mathrm{~A} \mathrm{a}$ & $6,44 \mathrm{~A}$ \\
\hline $\mathrm{CV}(\%)$ & 6,65 (temperatura) & 16,70 (contagem) & 11,16 \\
$\mathrm{dms}$ & 7,90 (temperatura) & 18,02 (contagem) & 1,20 \\
\hline
\end{tabular}

*Médias seguidas pela mesma letra maiúscula, em cada coluna e minúscula na linha, não diferem entre si pelo teste de Tukey a $5 \%$ de probabilidade.

Para avaliar a germinação de sementes de pinhãomanso em laboratório Dantas et al. (2009) sugerem semear as sementes em rolo de papel e mantê-las em germinador a $25{ }^{\circ} \mathrm{C}$, enquanto Martins et al. (2008) recomendam usar as temperaturas alternadas $20-30{ }^{\circ} \mathrm{C}$ em substrato de areia ou papel e com contagem final aos 10 dias da semeadura. Os dados obtidos demonstram que a germinação de sementes de pinhão-manso pode ser avaliada em substrato papel tanto na temperatura de $25{ }^{\circ} \mathrm{C}$ quanto na de $20-30{ }^{\circ} \mathrm{C}$, com contagem final aos 12 dias após semeadura.
Nas sementes desinfestadas de pinhão-manso (Figura 1) foi observada incidência dos fungos Alternaria sp., Cladosporium sp., Colletotrichum gloeosporioides, Epicoccum sp., Fusarium spp., Helminthosporium sp., Macrophomina sp., Nigrospora sp., Pestalotiopsis sp. e Phoma sp. Foi observada maior incidência de Cladosporium sp. (92\%) seguido dos gêneros Alternaria sp. (76\%), Fusarium spp. (62\%), Colletotrichum gloeosporioides (42,5\%), Epicoccum sp. (31\%), Macrophomina sp. (11\%), Nigrospora sp. (11\%), Helminthosporium sp. (9,5\%), Pestalotiopsis sp. (4,5\%) e Phoma sp. (3,5\%).

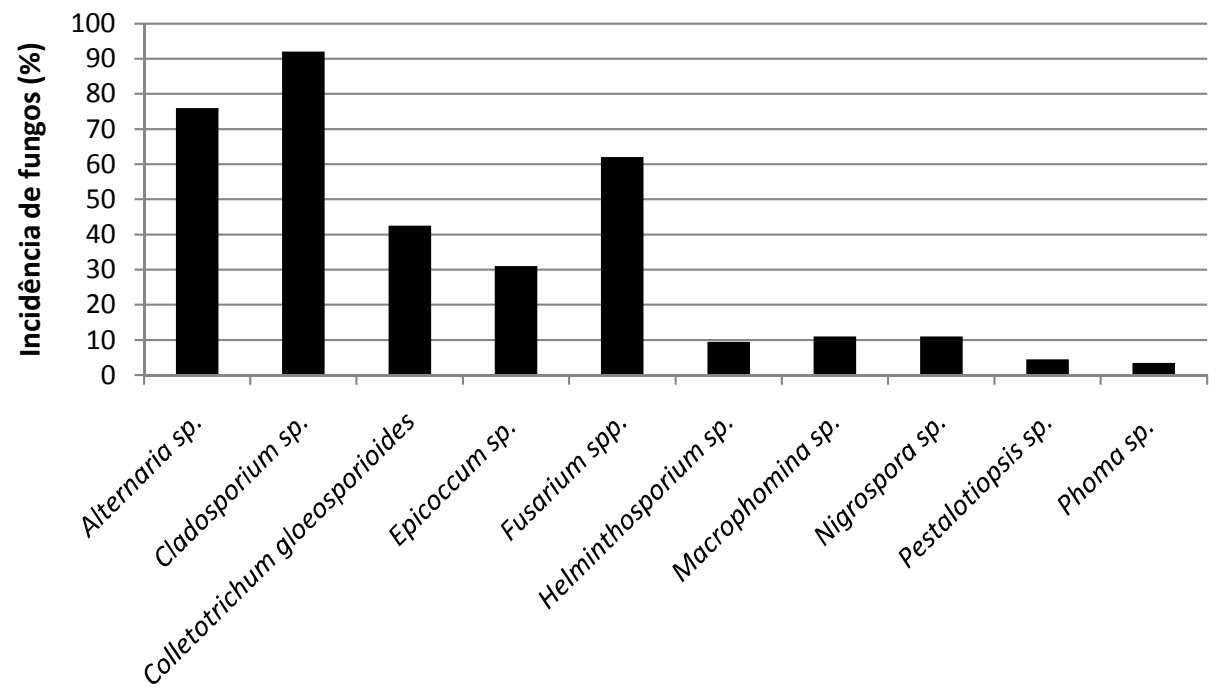

FIGURA 1. Incidência de fungos (\%) em sementes de Jatropha curcas previamente desinfestadas com hipoclorito de sódio.

Para as sementes sem desinfestação os fungos detectados (Figura 2) foram os mesmos das sementes desinfestadas tendo sido observada maior incidência de Cladosporium sp. (95\%) seguido de Fusarium spp. (75\%),
Alternaria sp. (65,5\%), Colletotrichum gloesporioides (35\%), Epicoccum sp. (26,5\%), Nigrospora sp. (11\%), Macrophomina sp. (7\%), Helminthosporium sp. (6\%), Pestalotiopsis sp. (5\%) e Phoma sp. (3,5\%). 


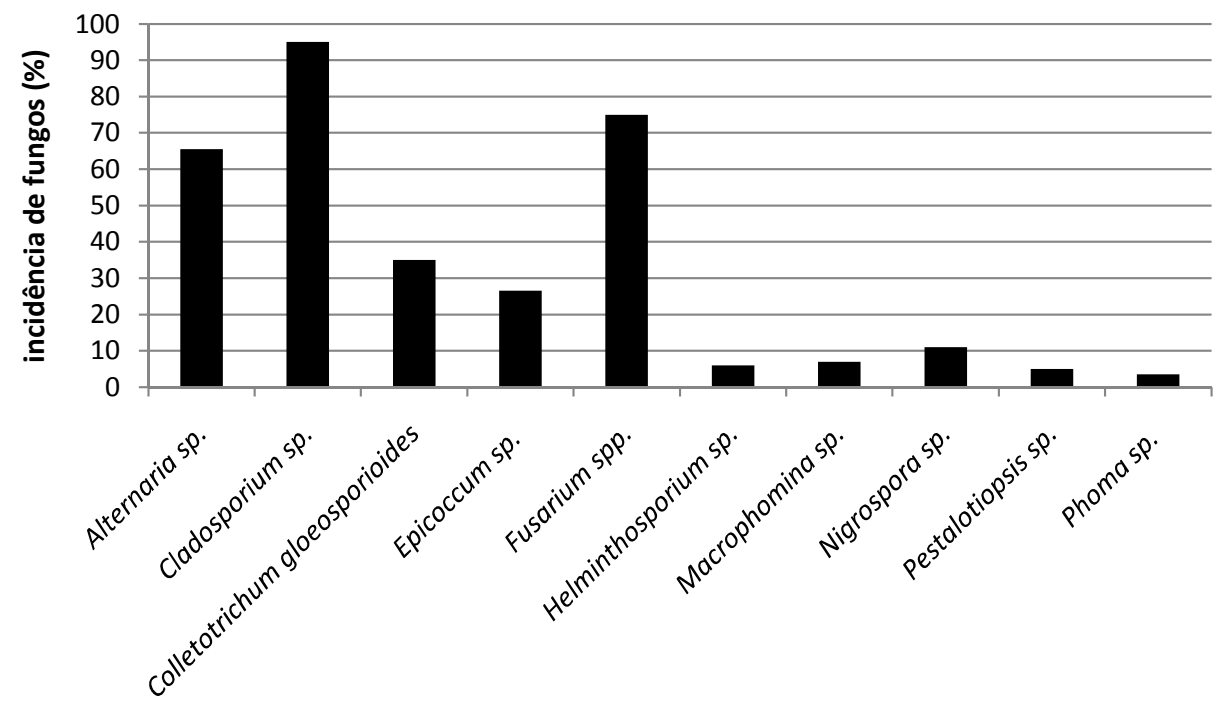

FIGURA 2. Incidência de fungos (\%) em sementes de Jatropha curcas sem desinfestação prévia.

No experimento não foi constatada diferença acentuada na incidência de fungos entre as sementes desinfestadas ou não com hipoclorito de sódio. Este resultado discorda daquele obtido por Melo et al. (2007) que observaram nas sementes desinfestadas de pinhãomanso menor incidência dos fungos pertencentes aos gêneros encontrados. A concentração de hipoclorito de sódio e o tempo de desinfestação podem não ter sido adequados ou a localização dos patógenos não possibilitou efeito do tratamento.

Dos fungos verificados neste trabalho, os pertencentes aos gêneros: Alternaria, Colletotrichum e Fusarium já foram relatados em sementes de pinhão-manso, em outros trabalhos (Melo et al., 2007; Moraes et al., 2009). Contudo, como foram avaliadas sementes recém colhidas, vários fungos de campo apresentaram alta incidência, enquanto não foi constatada a presença de fungos de armazenamento como Aspergillus spp., relatado por Melo et al. (2007) e Moraes et al. (2009), e Penicillium spp. (Melo et al., 2007).

Vários gêneros de fungos detectados nas sementes de pinhão-manso, como: Alternaria, Macrophomina, Cladosporium, Colletotrichum, Fusarium, Phoma e Helminthosporium são potencialmente patogênicos.

É importante destacar que o lote de sementes mesmo com essa alta incidência de fungos, de diferentes gêneros, apresentou germinação elevada, indicando que estes exerceram pouca ou nenhuma interferência na germinação. Este fato, à primeira vista tranquilizador, é na realidade preocupante, pois os fungos detectados são potencialmente patogênicos, podendo tornar as sementes importante fonte de disseminação de inóculo para diferentes regiões. Tendo em vista haver poucas informações sobre a ocorrência de doenças em pinhão-manso, estudos devem ser realizados visando identificar o potencial desses patógenos em causar doenças a essa cultura, bem como o papel das sementes na transmissão das mesmas.

\section{CONCLUSÕES}

A germinação de sementes de Jatropha curcas pode ser avaliada em substrato papel com contagem final aos 12 dias após semeadura tanto na temperatura de $25{ }^{\circ} \mathrm{C}$ quanto de $20-30{ }^{\circ} \mathrm{C}$;

As sementes recém-colhidas de pinhão-manso, independente da desinfestação superficial, apresentam incidência de fungos como Alternaria sp., Macrophomina sp., Cladosporium sp.; Colletotrichum gloeosporioides, Fusarium spp., Pestalotiopsis sp., Phoma sp., Helminthosporium sp., Epicoccum sp. e Nigrospora sp.

\section{REFERÊNCIAS}

ARRUDA, F.P.; BELTRÃO, N.E.M.; ANDRADE, A.P.; PEREIRA, W.E.; SEVERINO, L.S. Cultivo de pinhãomanso (Jatropha curcas L.) como alternativa para o semiárido nordestino. Revista de Oleaginosas e Fibrosas, v.8, p.789-799, 2004. 
BARNETT, H.C.; HUNTER, B.B. Illustrated genera of imperfect fungi. 3.ed. Minneapolis: Burgess Publications, 1987. $218 \mathrm{p}$.

BELTRÃO, N.E.M.; SILVA, L.C.; VASCONCELOS, O.L.; AZEVEDO, D.M.P.; VIEIRA, D.J. Fitologia. In: AZEVEDO, D.M.P.; LIMA, E.F. (Ed.). O agronegócio da mamona no Brasil. Campina Grande: Embrapa Algodão, 2001. p.36-61.

BRASIL. Ministério da Agricultura e Reforma Agrária. Secretaria Nacional de Defesa Agropecuária. Departamento Nacional de Defesa Vegetal. Coordenação de Laboratório Vegetal. Regras para análise de sementes. Brasília, DF, 1992. 365p.

BRASIL. Ministério da Agricultura, Pecuária e Abastecimento. Regras para análise de sementes. Ministério da Agricultura, Pecuária e Abastecimento. Secretaria de Defesa Agropecuária. Brasília, DF: Mapa/ ACS, 2009. 399p.

CARVALHO, N.M.; NAKAGAWA, J. Sementes: ciência, tecnologia e produção. 4.ed. Jaboticabal: Funep, 2000. $588 \mathrm{p}$.

DANTAS, B.F.; SILVA, F.F.S.; LOPES, A.P.; DRUMMOND, M.A. Tecnologia de sementes de pinhão manso (Jatropha curcas): avaliações iniciais da qualidade fisiológica. Disponível em:www.biodiesel.gov.br/docs/ congresso1007/agricultura/69.pdf. Acesso em: 15 jan. 2009.

MACHADO, J.C. Patologia de sementes: fundamentos e aplicações. Brasília, DF: MEC/ESAL/FAEPE, 1988. 106p.
MAGUIRE, J.D. Speed of germination-aid in selection and evaluation for seedling emergence and vigor. Crop Science, v.2, n.1, p.176-177, 1962.

MARTINS, C.C; MACHADO, C.G.; CAVASINI, R. Temperatura e substrato para o teste de germinação de sementes de pinhão-manso. Ciência e agrotecnologia, v.32, n.3, p.863-868, 2008.

MELO, M.F.V.; SANTOS, H.O.; SILVA-MANN, R., MESQUITA, J.B. Fungos associados a sementes de pinhão manso (Jatropha curcas L.). 2007. Disponível em: www.biodiesel.gov.br/docs/congresso2007/agricultura/46. pdf. Acesso em: 19 jul. 2009.

MORAES, A.F.G.; ALVES, E.; MORAES, W.; GIMENES, F.H.S.; CARVALHO, P.A. Ocorrência de fungos em sementes de pinhão manso (Jatropha curcas L.). Informativo ABRATES. Londrina, v.19, n.2, p.416, 2009.

NEERGAARD, P. Seed Pathology. London: Mac Millan, 1979. v.1, 839p.

SATURNINO, H.M.; PACHECO, D.D.; KAKIDA, J.; TOMINAGA, N.; GONÇALVES, N.P. Produção de oleaginosas para o biodiesel. Informe Agropecuário, v.26, p.44-74, 2005.

SUJATHA, M.; REDDY, T.P.; MAHASI, M.J. Role of biotechnological interventions in the improvement of castor (Ricinus communis L.) and Jatropha curcas L. Biotechnology Advances, v.26, p.424-435, 2008. 